\title{
P04-27. Monoclonal neutralizing antibodies inhibit HIV-I transfer from immature dendritic cells to human primary CD4 T-lymphocytes
}

\author{
K Xu, V Holl, M Peressin, T Decoville, S Schmidt, A Aubertin and C Moog*
}

Address: Institute of Virology, UMR INSERM/UDS, Strasbourg, France

* Corresponding author

from AIDS Vaccine 2009

Paris, France. 19-22 October 2009

Published: 22 October 2009

Retrovirology 2009, 6(Suppl 3):P55 doi:10.1 I86/I742-4690-6-S3-P55

This abstract is available from: http://www.retrovirology.com/content/6/S3/P55

(c) 2009 Xu et al; licensee BioMed Central Ltd.

\section{Background}

Dendritic cells (DCs) present on the mucosa site are considered as one of the first target cells of HIV-1 following sexual transmission. It has been demonstrated that immature DCs could transfer infectious HIV-1 particles to CD4 T-lymphocytes via a virological synapse in trans. The aim of this study was to investigate the capacity of monoclonal neutralizing antibodies to inhibit HIV-1 transfer from immature DCs to CD4 T-lymphocytes.

\section{Methods}

Immature DCs were generated from purified human blood monocytes. They were infected with HIV-1BaL for 2 hours and then washed extensively before exposure to purified primary PHA-activated CD4 T-lymphocytes in the presence or in the absence of monoclonal neutralizing antibodies. At different time-points, the percentage of HIV-infected DCs and CD4 T-lymphocytes were measured by flow cytometry detection of intracellular viral p24 antigen. The quantity of virus particles released in the supernatant was determined by p24 ELISA.

\section{Results}

In the presence of CD4 T-lymphocytes, we found a strong enhancement of the percentage of p24-positive immature DCs. Monoclonal neutralizing IgG1b12, 2F5, 2G12 or $4 \mathrm{E} 10$ added 2 hours after infection of immature DCs could totally inhibit HIV-1 replication in the CD4 T-lymphocytes. Nevertheless, due to the efficient HIV-1 replication in DCs in the presence of CD4 T-lymphocytes, HIV-1 p24 released in the supernatant of the co-culture was only reduced in presence of antibodies.

\section{Conclusion}

Here we clearly demonstrated that neutralizing antibodies are efficient inhibitors of HIV transfer to CD4 T lymphocytes and that HIV-1 replication becomes very efficient in DCs when they are co-cultured with primary CD4 T-lymphocytes. These results suggest that antibodies should thus be induced rapidly at the mucosal site to prevent DC infection and transfer to CD4 T-lymphocytes. 